\title{
A Rare Cause of Gastric Variceal Bleeding
}

\author{
Yesenia Ramos $^{a}$ Dorina Gui ${ }^{b}$ Eric Chak ${ }^{c}$ \\ aDepartment of Internal Medicine, University of California Davis School of Medicine, \\ Sacramento, CA, USA; bepartment of Pathology, University of California Davis School of \\ Medicine, Sacramento, CA, USA; 'Division of Gastroenterology and Hepatology, University \\ of California Davis School of Medicine, Sacramento, CA, USA
}

\section{Keywords}

Noncirrhotic portal hypertension · Oxaliplatin · Obliterative portal venopathy · Gastric variceal bleed

\section{Abstract}

A 68-year-old woman with stage III colon cancer status after right hemicolectomy and adjuvant FOLFOX (5-fluorouracil/leucovorin/oxaliplatin) chemotherapy was hospitalized for melena and found to have new-onset esophageal and gastric varices on esophagogastroduodenoscopy. Her workup did not reveal an underlying liver disease, but her liver biopsy showed noncirrhotic portal hypertension from obliterative portal venopathy (OPV). The development of OPV is likely from her use of oxaliplatin-based chemotherapy.

\section{Introduction}

Portal hypertension is defined as an increase in the portal-caval venous pressure gradient exceeding $5 \mathrm{~mm} \mathrm{Hg}$. The most common cause of portal hypertension in the Western world is cirrhosis, and $<10 \%$ of cases of portal hypertension are due to noncirrhotic portal hypertension (NCPH) [1]. NCPH can be classified as prehepatic, intrahepatic, and posthepatic. We present a case of a woman without a history of liver disease and a liver biopsy without evidence of cirrhosis who presented with gastric variceal hemorrhage secondary to intrahepatic NCPH.

\section{Karger"}


Fig. 1. The liver biopsy showed mild steatosis and mild portal inflammation. In most of the portal tracts, the portal veins appear narrowed or are difficult to recognize (hematoxylin \& eosin stain. $\times 200$ ).

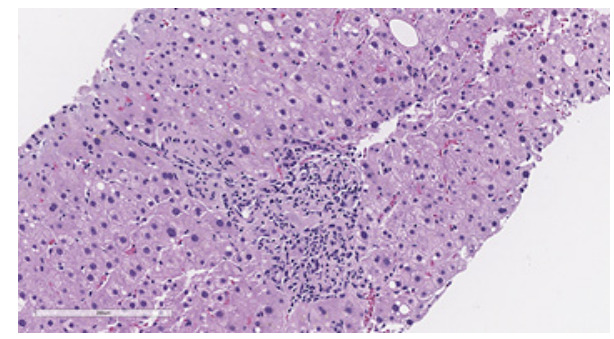

\section{Case Report}

A 68-year-old Cuban woman with a past medical history of colon cancer status after right hemicolectomy and adjuvant FOLFOX presented 2 years after completing her chemotherapy with melena. She underwent 12 cycles of a modified FOLFOX6 regimen that included leucovorin calcium, fluorouracil, and oxaliplatin. Since her chemotherapy, she developed moderate thrombocytopenia and initially had mild AST/ALT elevation which later normalized. To further workup her thrombocytopenia, she had a CT abdomen which showed splenomegaly and an incidental finding of distal esophageal and short gastric axis varices. She had a Fibroscan which showed no significant fibrosis or steatosis. Before she could make it to her outpatient hepatology referral, she developed significant melena and was hospitalized.

On arrival to the hospital, she had multiple episodes of melena and hemodynamic instability. An esophagogastroduodenoscopy revealed small esophageal varices and a large irregular isolated gastric varix (IGV1) in the fundus with red wale signs. Therefore, an urgent balloon-occluded retrograde transvenous obliteration (BRTO) was used to treat her gastric varices.

The cause of her gastric and esophageal varices was unknown. There was no known history or family history of liver disease. Her only risk factors for liver disease were being overweight and prior chemotherapy. The patient's outpatient medications included levothyroxine, lasix, potassium, pyridoxine, and prior use of milk thistle supplements. She had no significant alcohol use. Her labs were notable for normocytic anemia, moderate thrombocytopenia, and normal liver tests and synthetic liver function. A transthoracic echocardiogram demonstrated normal left ventricular systolic function with an ejection fraction of $65 \%$ and normal right ventricular function.

She underwent an extensive workup for chronic liver disease. Her serologies were negative for viral hepatitis, ANA negative, SMAB negative, mitochondrial Ab negative, ceruloplasmin normal, A1AT elevated, and ferritin negative. Stool examination was negative for schistosomiasis. Her hepatic venous pressure gradient was $2 \mathrm{~mm} \mathrm{Hg}$ with a $15 \mathrm{~mm} \mathrm{Hg}$ hepatic vein wedge pressure and $13 \mathrm{~mm} \mathrm{Hg}$ hepatic vein free pressure of the right hepatic vein after the BRTO. She had clear signs of portal hypertension including splenomegaly and varices that required BRTO, and her imaging was negative for portal, hepatic, and splenic vein thrombosis. The transjugular needle core liver biopsy showed mild steatosis and mild portal inflammation. In most of the portal tracts, the portal veins appear narrowed or are difficult to recognize (shown in Fig. 1). No features of cirrhosis, bridging fibrosis, or nodular regenerative hyperplasia are evident in the biopsy (shown in Fig. 2); therefore, these findings were suggestive of obliterative portal venopathy.

She has since completed surveillance for colon cancer and is no longer receiving yearly CT scans. Based on expert consensus, surveillance of IGV1 is not required after BRTO [2].

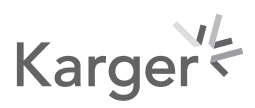


Fig. 2. The liver biopsy had no features of cirrhosis, bridging fibrosis, or nodular regenerative hyperplasia (trichrome stain. $\times 300$ ).

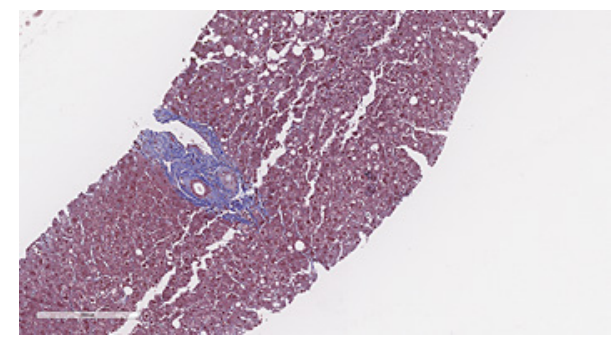

\section{Discussion}

NCPH can be classified as prehepatic, intrahepatic, and posthepatic based on the site of obstruction in the hepatic circulation. Prehepatic causes include splenic and portal vein thrombosis, extrinsic compression of splenic or portal vein, and arteriovenous fistula. However, posthepatic causes include Budd-Chiari syndrome, IVC thrombus, constrictive pericarditis, and right-sided HF [1, 3]. Our patient's imaging did not reveal vascular thrombosis or extrinsic compression of the splenic or portal vein. Her transthoracic echocardiogram did not show heart failure or constrictive pericarditis. Therefore, she has intrahepatic NCPH.

Intrahepatic causes of NCPH are subcategorized as presinusoidal, sinusoidal, and postsinusoidal. Presinusoidal intrahepatic causes of NCPH occur when there is obstruction at the portal vein at the level of the portal tracts. Some causes include schistosomiasis, polycystic disease, small duct sclerosing cholangitis, primary biliary cholangitis, and granulomatous disease secondary to sarcoidosis or tuberculosis $[1,3]$. Our patient's laboratory, imaging, and liver biopsy did not suggest these conditions. Her liver biopsy showed obliterative portal venopathy which is another cause of presinusoidal intrahepatic NCPH. Sinusoidal intrahepatic obstruction within the sinusoidal lumen or in the space of Disse can be caused by amyloidosis, steatohepatitis, nodular regenerative hyperplasia, congenital hepatic fibrosis, sinusoidal fibrosis from toxins, and medications including methotrexate [1,3]. From these, her liver biopsy showed mild steatosis. Last, an injury to the sinusoidal cells in zone 3 where the sinusoids communicate with central veins would be categorized as an intrahepatic postsinusoidal cause of NCPH, which can be seen in sinusoidal obstructive syndrome [3].

Our patient has biopsy-proven obliterative portal venopathy as the cause of her intrahepatic NCPH. Oxaliplatin with a fluoropyrimidine has been standard adjuvant therapy in patients with stage III colon cancer [4]. A case report in 2012 attributed a patient's symptomatic portal hypertension from hepatoportal sclerosis, now known as obliterative portal venopathy, to his use of oxaliplatin [5]. Subsequent reports had similar conclusions. In one case series, 6 patients with colorectal cancer who were treated with oxaliplatin developed thrombocytopenia, splenomegaly, and varices. Out of these 6 patients, 2 had variceal hemorrhage [6]. These case studies noted a possible association between the use of oxaliplatinbased chemotherapy and the development of intrahepatic NCPH. Oxaliplatin-associated NCPH has an estimated incidence rate from 19\% to 52\% [7]. Morioka et al. [8] conducted a literature review using key words "oxaliplatin" and "portal hypertension" which yielded 27 articles on PubMed on April 2020. Of these articles, 4 of them described radiological and/or surgical interventions for the oxaliplatin-based chemotherapy associated portal hypertension. A total of 31 cases required interventions including 25 partial splenic embolization, 4 splenic arterial ligation, 1 transjugular intrahepatic portosystemic shunt (TIPS), and 1 embolization of the peristomal varices [8]. From these interventions, the TIPS procedure was the only one done for esophageal variceal bleeding. Given the limited literature on oxaliplatin-associated NCPH, further research is warranted especially since variceal bleeds can be life threatening.

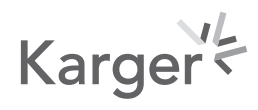


Fig. 3. Proposed treatment algorithm for acute gastric variceal hemorrhage. Red line indicates in case of failure of band ligation or cyanoacrylate glue injection then the next step would be salvage therapy. EV, esophageal varices; GOV, gastroesophageal varices; IGV, isolated gastric varix; TIPS, transjugular intrahepatic portosystemic shunt; BRTO, balloonoccluded transvenous obliteration.

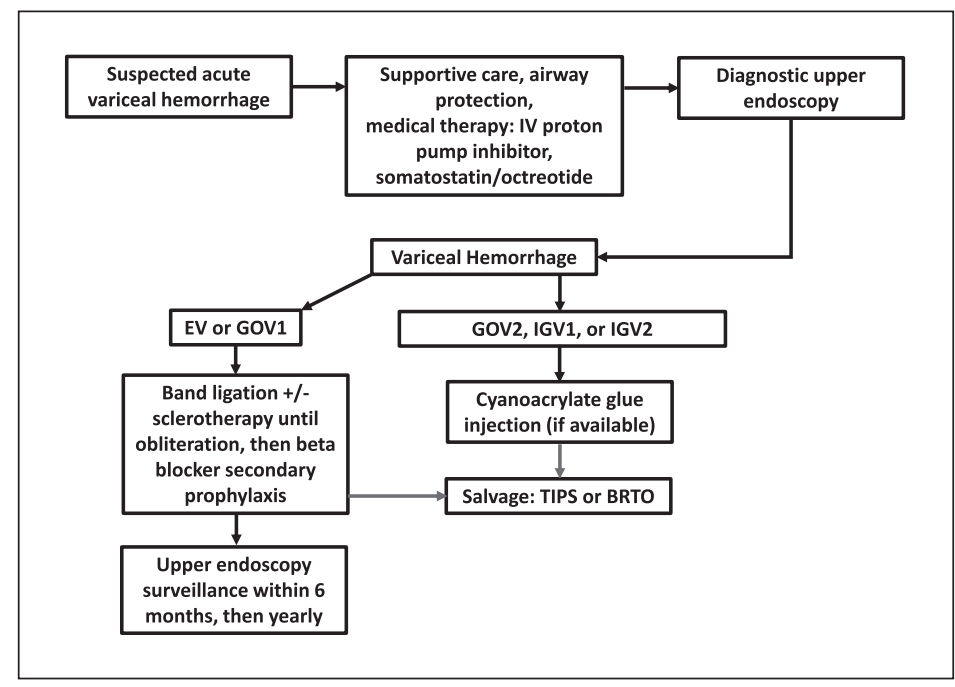

Sarin et al. [9] describes a general medical and endoscopic management of acute gastric variceal bleeding in NCPH. Gastroesophageal varices are extension of esophageal varices that extend below the gastroesophageal junction along the lesser curvature (GOV1) or into the fundus of the stomach (GOV2). Our patient had an isolated gastric varix that was in the fundus of the stomach (IGV1), while IGV2 refers to ectopic varices located anywhere in the stomach [9]. Regardless of where the gastric varices are located, the first step is to ensure hemodynamic stability and airway protection. Vasoactive drugs such as somatostatin or octreotide are used followed by upper gastrointestinal endoscopy. Depending on location of the varices, gastric variceal bleeding can be controlled with endoscopic band ligation, sclerotherapy, or cyanoacrylate glue injection (shown in Fig. 3). About 10\% of cases need rescue therapies including TIPS, BRTO, devascularization, or a decompression shunt [2]. The use of $\beta$-blockers for secondary prophylaxis is recommended with GOV1 but not for GOV2 or IGV1 since clinical trials have shown no benefit to $\beta$-blockers in this setting [10].

Clinical screening for portal hypertension should be done in patients who have been treated with oxaliplatin. Oxaliplatin has been associated with splenomegaly and thrombocytopenia. A study suggested that increases in spleen size correlated with increasing grade of intrahepatic injury and could serve to identify patients with possible oxaliplatin toxicity [11]. Therefore, patients who have a history of oxaliplatin-based chemotherapy and signs of portal hypertension should be considered for varices screening to determine their management.

\section{Acknowledgment}

This manuscript does not include any nonauthor contributors to acknowledge.

\section{Statement of Ethics}

This case report complies with the guidelines for human studies and was conducted ethically in accordance with the World Medical Association Declaration of Helsinki. This study protocol was reviewed and the need for approval was waived by the Ethics Committee at the University of California, Davis. Informed written consent to publish this case including pathology images was obtained from the patient. 


\section{Conflict of Interest Statement}

The authors have no conflicts of interest to declare.

\section{Funding Sources}

No funding was received for this study.

\section{Author Contributions}

Yesenia Ramos wrote the manuscript. Dorina Gui and Eric Chak reviewed the manuscript. All authors approved the final version of the manuscript.

\section{Data Availability Statement}

All data generated or analyzed during this study are included in the article. Further enquiries can be directed to the corresponding author.

\section{References}

1 Garcia-Tsao G. Idiopathic noncirrhotic portal hypertension: what is it? Clin Liver Dis. 2015 May;5(5):120-2.

2 Sarin SK, Philips CA, Khanna R. Noncirrhotic portal hypertension: medical and endoscopic management. Clin Liver Dis. 2015 Nov;6(5):107.

3 Nakhleh RE. The pathological differential diagnosis of portal hypertension. Clin Liver Dis. 2017 Sep;10(3): 57-62.

4 Slade JH, Alattar ML, Fogelman DR, Overman MJ, Agarwal A, Maru DM, et al. Portal hypertension associated with oxaliplatin administration: clinical manifestations of hepatic sinusoidal injury. Clin Colorectal Cancer. 2009 Oct; $8(4): 225-30$.

5 Lawal TO, Farris AB, El-Rayes BF, Subramanian RM, Kim HS. Oxaliplatin-induced hepatoportal sclerosis, portal hypertension, and variceal bleeding successfully treated with transjugular intrahepatic portosystemic shunt. Clin Colorectal Cancer. 2012 Sep;11(3):224-7.

6 Taieb J, Gallois C. Adjuvant chemotherapy for stage III colon cancer. Cancers. 2020 Sep;12(9):2679.

7 Gioia S, Di Martino M, Minozzi M, Nardelli S, Cortesi E, Riggio O. Incidence of portal hypertension in patients exposed to oxaliplatin. Dig Liver Dis. 2019 Sep 1;51(9):1348-50.

8 Morioka D, Izumisawa Y, Yamaguchi K, Sato K, Komiyama S, Nakagawa K, et al. Surgical intervention for portal hypertension caused by oxaliplatin-based chemotherapy: a case report and a review of literature regarding radiological and/or surgical interventions for oxaliplatin-associated portal hypertension. Clin J Gastroenterol. 2020 Oct;13(5):799-805.

9 Sarin SK, Lahoti D. Management of gastric varices. Baillieres Clin Gastroenterol. 1992 Sep 1;6(3):527-48.

10 Hung HH, Chang CJ, Hou MC, Liao WC, Chan CC, Huang HC, et al. Efficacy of non-selective $\beta$-blockers as adjunct to endoscopic prophylactic treatment for gastric variceal bleeding: a randomized controlled trial. J Hepatol. 2012 May 1;56(5):1025-32.

11 Overman MJ, Maru DM, Charnsangavej C, Loyer EM, Wang H, Pathak P, et al. Oxaliplatin-mediated increase in spleen size as a biomarker for the development of hepatic sinusoidal injury. J Clin Oncol. 2010 May;28(15): 2549-55.

\section{Karger' ${ }^{\prime}$}

